\title{
1 Bioelectrochemical reduction of an azo dye by a Shewanella oneidensis
}

\section{$2 \quad$ MR-1 formed biocathode}

3

4 Shu-Hong Gao ${ }^{1,2 \S}$, Lai Peng ${ }^{2,4 \S}$, Yiwen Liu $^{4}, \mathrm{Xu}$ Zhou $^{2}$, Bing-Jie-Ni $^{2}$,Philip L Bond ${ }^{2}$, Bin

$5 \quad$ Liang $^{3 *}$, Ai-Jie Wang ${ }^{1,3^{* *}}$

$6{ }^{1}$ State Key Laboratory of Urban Water Resource and Environment, Harbin Institute of

7 Technology, Harbin 150090, PR China

$8{ }^{2}$ Advanced Water Management Centre, The University of Queensland, St. Lucia, Brisbane,

9 QLD 4072, Australia

$10{ }^{3}$ Key Laboratory of Environmental Biotechnology, Research Center for Eco-Environmental

11 Sciences, Chinese Academy of Sciences, Beijing 100085, PR China

$12{ }^{4}$ Centre for Microbial Ecology and Technology, Faculty of Bioscience Engineering, Ghent 13 University, Ghent 9000, Belgium

15 Correspondence: [Dr. Bin Liang, Key Laboratory of Environmental Biotechnology, Research 16 Center for Eco-Environmental Sciences, Chinese Academy of Sciences, Beijing 100085, PR 17 China].

$18 \quad$ E-mail: [binliang@ @rcees.ac.cn]

19

20 Correspondence: [Prof. Ai-Jie Wang, State Key Laboratory of Urban Water Resource and

21 Environment, Harbin Institute of Technology, Harbin 150090, PR China].

22 E-mail: [waj0578@hit.edu.cn; ajwang@ rcees.ac.cn]

23 §. Gao and L. Peng contributed equally to this work.

24

25

26 


\section{Abstract}

28 Presently there is great interest to develop pure culture cathodes in bioelectrochemical systems (BES) for achieving decolorization/reduction of azo dyes. In this study, we

30 investigated the decolorization of a model azo dye, acid orange 7 (AO7) in a biocathode 31 inoculated with a model electrogenic microorganism Shewanella oneidensis MR-1 32 (MR-1). The decolorization efficiency of AO7 reached 95.8 $\pm 4.6 \%, 86.4 \pm 2.0 \%$, and $3377.8 \pm 1.0 \%$ in $46 \mathrm{~h}$ in the biocathode fed with lactate, in the biocathode without lactate, 34 and in the abiotic cathode respectively. Thus, enhanced decolorization of AO7 occurred 35 in the biocathode, compared to the abiotic cathode, regardless of the presence of lactate. 36 To further investigate the AO7 reduction mechanism and electron transfer between the 37 electrode and S. oneidensis, the cathode potential was controlled such that hydrogen 38 was not produced. In the absence of lactate, the AO7 decolorization efficiency $(78.5 \pm 0.8 \%)$ and sulfanilic acid (SA) production efficiency $(70.9 \pm 1.5 \%)$ in the biocathode were higher than the AO7 decolorization efficiency $(63.7 \pm 3.6 \%)$ and the SA production $(56.1 \pm 1.9 \%)$ efficiency in the abiotic cathode. This suggests that the reduction of $\mathrm{AO}$, with electrode as the sole electron donor, was enhanced by electron transfer between the cathode and the $S$. oneidensis formed biofilm on the cathode surface. This study demonstrated that $S$. oneidensis enhanced the capture of electrons from the cathode electrode for the reduction of the organic pollutant AO7.

47 Keywords: AO7 reduction; biocathode; Bioelectrochemical systems; Shewanella 48 oneidensis MR-1

\section{1, Introduction}


51 Azo dyes are compounds with aromatic structures characterized by containing one or 52 more azo linkages $(-\mathrm{N}=\mathrm{N}-)$ and they are widely used in the textile, leather, cosmetics, 53 and food industries (Elisangela et al. 2009, Tony et al. 2009). Azo dyes can be water or 54 oil soluble, and the presence of a sulfonated group results in the compounds being 55 highly water-soluble. Consequently, azo dyes containing these groups are easily 56 mobilized into water systems during production and applications (Tan et al. 2005).

57 Environmental contamination by these azo dyes even at very low concentrations can 58 cause water streams to become highly colored and give rise to undesirable aesthetic 59 effects (Pandey et al. 2007, Lončar et al. 2012, Singh et al. 2015).

60

61 Azo dyes are generally persistent under aerobic conditions (Tan et al. 2013). However, under anaerobic conditions azo dyes can be reduced and decolorized, with the breakdown of azo bonds producing products that are easily mineralized (Tan et al. 2005,

64 Wang et al. 2011). This anaerobic decolorization is a cost efficient process; however, it 65 is usually very slow and requires an organic co-substrate to provide electrons for the 66 reduction (dos Santos et al. 2005). The addition of the co-substrate adds to the costs and 67 unwanted methane production since the dosage typically far exceeds the stoichiometric requirements (Van der Zee and Villaverde 2005). Thus, treatment of wastewater

69 containing azo dyes is a challenging issue that requires improvement. Acid orange 7 70 (AO7) is an azo dye that is widely used in the textile, food and cosmetics industries, 71 thus it is a significant component of wastewaters produced by these manufacturers that 72 requires removal (Mu et al. 2009). 
74 Recently, wastewater treatment capabilities of bioelectrochemical systems (BES) have 75 being extensively explored for degradation of a number of organic and inorganic 76 contaminates (Wang et al. 2015). Contaminants such as nitroaromatics (Wang et al. 77 2011, Liang et al. 2014, Feng et al. 2015), azo dyes (Liu et al. 2015), and halogenated 78 aromatics (Liang et al. 2013, Kong et al. 2014) can be reductively eliminated under 79 anaerobic conditions with acclimated biocathode communities. However, the biological 80 reduction mechanisms of the pollutants at the biocathode are unclear and such details 81 are required for optimization of the treatment processes. Bacterial reduction of azo dyes 82 has been investigated using various pure cultures under cathodic conditions while 83 adding specific carbon sources. Mostly these pure cultures are of the Shewanella genus 84 and include Shewanella decolorationis S12 (Hong et al. 2007, Hong and Gu 2010, Yang 85 et al. 2015), Shewanella sp. J18143 (Pearce et al. 2006), S. oneidensis (Pearce et al. 2006) as well as Pseudomonas sp. WYZ-2 (Wang et al. 2014). However, mostly these

87 studies focused on the performance of the azo dye removal and the BES conditions 88 rather than on determining details of the cathode electron transfer mechanisms for the 89 reduction of the dye.

90

$91 S$. oneidensis exhibits very diverse respiratory capabilities. In addition to aerobic 92 respiration it can conduct anaerobic respiration using a range of electron acceptors that 93 include fumarate (Rosenbaum et al. 2011), nitrobenzene (Cai et al. 2012), Cr(VI) 94 (Belchik et al. 2011), Fe(III) citrate (Rosenbaum et al. 2012), hexahydro-1,3,5-trinitro95 1,3,5-triazine (RDX) (Perreault et al. 2012) as well as azo dyes such as Naphthol Green 96 B (Xiao et al. 2012). As a consequence of these diverse respiratory capabilities, 
97 Shewanella species are often used in electrogenic studies for the reduction of organic 98 pollutants in BES (Rosenbaum et al. 2012).

100 In this study, a dual chamber BES was used for the removal of azo dye AO7 at a 101 biocathode in the presence of S. oneidensis. Removal efficiency of AO7 was compared 102 between biocathodes in the presence and absence of the co-substrate lactate, as well as 103 between the biocathode and the abiotic cathode. This work supports the feasibility of 104 AO7 reduction mediated by the cathodic biofilm of $S$. oneidensis. To our best 105 knowledge, this is the first report to demonstrate that S. oneidensis MR-1 enhances 106 cathodic electron capture for the reduction of the organic pollutant AO7.

\section{Materials and methods}

\subsection{Anode and cathode inoculum}

110 The anodic microbial community was enriched from domestic wastewater that was used

111 to inoculate to the BES as previously described (Wang et al. 2011). Sodium acetate was

112 added to the anodic medium to ensure excess electron donor. A pure cultures of $S$. 113 oneidensis was used to form biofilms in the cathodic chamber. S. oneidensis from 114 glycerol stocks was firstly incubated in Luria-Bertani (LB) medium at $30^{\circ} \mathrm{C}$, shaken at $115150 \mathrm{rpm}$ overnight. One loop of bacterial suspension was then streaked onto a LB plate 116 and incubated overnight at $30^{\circ} \mathrm{C}$. From the plate, one pink colony was transferred to 117 liquid LB medium and incubated at $30^{\circ} \mathrm{C}$, shaken at $150 \mathrm{rpm}$ overnight. This suspension 118 was used as the BES cathode inoculum. The cathode medium consisted of a nutrient 119 solution that included Wolf's vitamins and trace elements (Wang et al. 2011). 120 Additionally, when included in the cathode medium batch tests, lactate was added at 10 
$121 \mathrm{mM}$ and $\mathrm{AO} 7$ was added at $0.28 \mathrm{mM}$ in the presence of lactate and $0.147 \mathrm{mM}$ in the

122 absence of lactate. The cathodic medium was prepared by boiling and purging with $\mathrm{N}_{2}$

123 gas (99.9\% purity) for $20 \mathrm{~min}$ before autoclaving at $121^{\circ} \mathrm{C}$ for $30 \mathrm{~min}$.

124

\section{$125 \quad 2.2$ BES reactor set up}

126 Dual chamber polycarbonate BES reactors were constructed and assembled, using 127 graphite fiber brush and carbon cloth as the anode and cathode materials respectively, as 128 previously described (Liang et al. 2013). The working volume of each chamber was 85

$129 \mathrm{~mL}$. The carbon cloth was immersed in $1 \mathrm{M}$ hydrochloric acid for $24 \mathrm{~h}$ and then in 130 deionized water for another $24 \mathrm{~h}$ before use. The assembled reactors, excluding the 131 carbon cloth, were covered with aluminum foil and autoclaved at $121^{\circ} \mathrm{C}$ for 30 min. 132 Following that, the carbon cloth was assembled into the BES in sterile conditions before 133 starting the batch experiments. An external power of $0.3 \mathrm{~V}$ was applied to assist the 134 AO7 reduction in the abiotic and biotic cathodes, unless otherwise mentioned.

136 All the BES reactors had the same anode conditions. For one set of reactors the cathode 137 was inoculated with S. oneidensis while in another set there was no inoculum and the 138 cathode was abiotic. The cathode inoculum was centrifuged at $5000 \mathrm{rpm}$ for $3 \mathrm{~min}$, the 139 supernatant was discarded, and then the pellet was dissolved in sterile cathode medium 140 before transferring it to the cathode chamber. This was incubated under anaerobic 141 conditions for $48 \mathrm{~h}$ to allow biofilm to develop on the cathode. Subsequently, the 142 cathode liquid was removed and the cathode chamber was replenished with another 143 batch of freshly prepared MR-1 inoculum and cathode medium. This procedure was 
144 repeated six times to achieve adequate biofilm development on the cathode surface 145 (Liang et al. 2013).

146

147 Two BES reactors with well-developed cathode biofilms were operated for three 148 repeated batch experiments under particular conditions with $\mathrm{AO} 7$ as the electron 149 acceptor. For each batch experiment fresh media was placed in the anode and cathode 150 chambers and the BES reactions were monitored. Each batch experiment lasted 151 approximately $60-80 \mathrm{~h}$ and was terminated when the current had nearly stopped 152 decreasing (closed circuit mode). For each reactor, the initial batch experiments were 153 operated in closed circuit mode, and then further batch tests were performed with the 154 two reactors switched to opened circuit, thus operated without external electron supply. 155 Another two BES reactors were operated as abiotic cathodes for three cycles initially 156 with closed circuit, and then as open circuit mode. A summary of the overall batch test 157 experiment design is provided in the results. All the BES batch experiments were 158 performed at room temperature $\left(26 \pm 2^{\circ} \mathrm{C}\right)$.

\section{$160 \quad 2.3$ Chemicals analysis}

161 During the batch experiments samples taken from the cathode were immediately filtered 162 through a $0.22 \mu \mathrm{m}$ filter, and AO7 concentrations were measured using UV-vis 163 spectrophotometer (Cary 50, Varian Inc., Australia) at $485 \mathrm{~nm}$ (Mu et al. 2009) over the 164 wavelength range of 200 to $750 \mathrm{~nm}$. The reduced product sulfanilic acid (SA) of AO7 165 was quantified by HPLC. The reverse-phase HPLC system (model e2695, Waters Co., 166 Milford, MA) was equipped with a Semmetry C18 column $(5 \mu \mathrm{m} ; 5 \times 250 \mathrm{~mm}$, Waters 
167 Co., USA) and a UV detector was used for SA measurement at $254 \mathrm{~nm}$ as described 168 previously (Mu et al. 2009).

169

\subsection{Cyclic Voltammetry analysis and SEM detection}

171 The cyclic voltammetry (CV) analysis was conducted using an electrochemical 172 workstation (model-660D, CH Instruments Inc., Austin, TX, USA) equipped with a 173 three-electrode system. Cyclic voltammograms were recorded with the scan rate of 5

$174 \mathrm{mV} \mathrm{S}^{-1}$. The abiotic cathode/biocathode and bioanode of the BES reactors were 175 designated as the working and counter electrodes, respectively. The saturated calomel 176 reference electrode (SCE, 0.247 V vs standard hydrogen electrode (SHE), model-217, 177 Shanghai Precise. Sci. Instru. Co., Ltd., China) was inserted into the cathode chamber. 178 Concentrations of $\mathrm{AO} 7$ and lactate were $0.147 \mathrm{mM}$ and $10 \mathrm{mM}$, respectively. All the 179 cathode potentials were reported against the SHE. The biocathode biofilms were 180 visualized by scanning electron microscopy (SEM) (HELIOS Nanolab 600i, Hillsboro, 181 OR, USA) at $20 \mathrm{kV}$ as described elsewhere (Xie et al. 2011).

182

\section{3. Results and Discussion}

1843.1 Biocathode formation in the dual-chamber BES

185 Prior to the AO7 reduction experiments biofilms were formed on the cathodes over 186 about a 12 day period by repeatedly inoculating the BES. During this biocathode 187 biofilm formation process, the current of the BES reactors increased over time (Fig. 188 1A). After 12 days the current had increased from around $2.0 \mathrm{~mA}$ to be stable at 4.7 $189 \mathrm{~mA}$, indicating the occurrence of a stable MR-1 biofilm on the cathodic electrode (Fig. 190 1A). This was evident by scanning electron microscopy images of the cathode in the 
191 absence of lactate after the biofilm formation period (Fig. 2 A\&B). The biofilm on

192 cathode is not quite thick due to at least two possible reasons: 1) all the experiments

193 were conducted under strictly anaerobic conditions which produce much less biomass

194 than that of aerobic conditions; 2) electrode served as the potential sole electrode donor

195 and inorganic bicarbonate as the carbon source in this study.

196

197 For the last two periods of catholyte replacement, further inoculum of S. oneidensis was not used. As expected AO7 (absorbance at $484 \mathrm{~nm}$ ) and SA (absorbance at $254 \mathrm{~nm}$ ) were not detected at the end of batch test (Fig. 1B), indicating the reduction of AO7 in 200 cathode chamber.

\subsection{Reduction of $A O 7$ in the BES}

203 The batch experiments for BES AO7 reduction were conducted in three groups: Group 204 I: AO7 removal in the presence of the co-substrate lactate; Group II: AO7 removal in 205 the absence of lactate; and Group III: AO7 removal without lactate with the cathode 206 potential held at $-408 \mathrm{mV}$ to avoid hydrogen production (vs. SHE). It was seen that current was produced in the batch experiments in the presence or absence of lactate (Fig. $3 \mathrm{~A}$ and B). However it was higher and more rapidly declined in the presence of 209 lactate. The reduction in current during the batch tests (Fig. 3) was likely due to the 210 decreasing levels of $\mathrm{AO} 7$ as it was being reduced. Electrode potentials and current 211 generation of cathode were recorded to evaluate the performance of the BES reactors 212 (Fig. 3). The cathode potentials were almost the same for the biocathode and abiotic cathode (Fig. 3A and B). This was possibly due to the similar conditions in anode

214 chamber which resulted in a similar anode potential, then similar cathode potentials 
215 occurred with the same applied extra power voltage. Nevertheless, current generation in 216 the closed circuit biocathode in the presence of lactate was higher than that of the closed

217 circuit biocathode in the absence of lactate (Fig. 3A and B). The higher the current in 218 the closed circuit biocathode, the more efficient the electrons transfer from electrons to 219 S. oneidensis, and the more efficient the AO7 reduction.

In Group I batch experiments there were three modes of reactor operation: (i)

222 biocathode BES operated with a closed circuit and an external power source of $0.3 \mathrm{~V}$

223 (this called the closed circuit biocathode), (ii) biocathode BES operated as an opened 224 circuit (opened circuit biocathode), and (iii) BES having an abiotic cathode with a 225 closed circuit and an external power source of $0.3 \mathrm{~V}$ (abiotic cathode). For all three 226 modes, the initial cathode $\mathrm{AO} 7$ and lactate concentrations were 0.285 and $10 \mathrm{mM}$, 227 respectively.

229 In the batch experiments the reduction of $\mathrm{AO} 7$ was observed in all three modes with the 230 highest removal obtained in the closed circuit biocathode (Fig. 4 A, C and E). The removal efficiency of AO7 in the closed circuit biocathode, opened circuit biocathode and abiotic cathode were $95.8 \pm 4.7 \%, 73.0 \pm 5.9 \%$ and $77.8 \pm 1.0 \%$, respectively after $46 \mathrm{~h}$

233 incubation. The removal efficiency of AO7 in the abiotic cathode was higher than that 234 of the opened circuit biocathode, indicating that the electron supply from the anode and applied external power supply of $0.3 \mathrm{~V}$ did promote the AO7 reduction (Fig. 4A). SA is a product of $\mathrm{AO} 7$ reduction (Bay et al. 2014) and its production was evident during the 237 removal of $\mathrm{AO} 7$ (Fig. 1B), consequently supporting that $\mathrm{AO} 7$ reduction occurred in the 238 BES. SA was detected in all three modes (closed circuit biocathode, opened circuit 
239 biocathode and abiotic cathode) (Fig. 4B), and its production was inversely correlated 240 with AO7 level. So the production rate of SA in the biocathode was much higher than

241 the opened circuit and the abiotic cathodes. The production efficiency of SA in the 242 closed circuit biocathode, opened circuit biocathode and abiotic cathode at $46 \mathrm{~h}$ were $24395.2 \pm 0.6 \%, 60.1 \pm 5.9 \%$ and $41.7 \pm 18.5 \%$, respectively. The removal efficiency of AO7 244 was consistent with the production efficiency of SA, this also indicating that there was 245 no consumption of SA in the biocathode when lactate was supplied. Moreover, the 246 kinetic constants under different operating conditions were further compared to 247 determine whether the biocathode enhanced AO7 reduction due to the fact that different 248 initial AO7 concentration was applied for Group I (in the presence of lactate) and Group 249 II \&III (in the absence of lactate) experiment (Table 1). The kinetic constant for closed 250 circuit biocathode of Group I was $0.063 \pm 0.002$, which was higher than $0.0479 \pm 0.0035$ 251 in closed circuit biocathode of Group II. However, the kinetics constant for closed 252 circuit abiotic cathode was comparable in Group I and Group II, which was $2530.0272 \pm 0.0027$ and $0.0324 \pm 0.002$ respectively, although the initial AO7 concentration 254 was different. Also it is obvious that the kinetics constant $(0.0479 \pm 0.0035)$ of 255 biocathode in Group II was higher than that $(0.0324 \pm 0.002)$ of abiotic cathode in Group 256 II. In conclusion, the presence of lactate enhanced the AO7 reduction in the cathode 257 chamber and AO7 could be more efficiently reduced in the biocathode rather than in the 258 abiotic cathode.

260 The electrons for the AO7 reduction by the MR-1 biocathode in Group I were provided 261 by the anode and the lactate in the catholyte. In contrast, the AO7 removal and SA 262 production in both the opened circuit biocathode and the abiotic cathode were much 
263 lower than that detected for the closed circuit biocathode (Fig. 4A and B), thus, 264 indicating that the biofilm was critical for the BES enhanced reduction of AO7.

266 To further investigate whether $S$. oneidensis could enhance the reduction of AO7 with

267 the cathode as the sole electron donor, BES batch experiments were performed in the 268 absence of lactate in the cathode (Group II). Two different modes were tested for 269 comparison in Group II: the biocathode and the abiotic cathode. For both modes, the 270 initial $\mathrm{AO} 7$ concentration was around $0.14 \mathrm{mM}$ and this was reduced during the BES 271 test, while simultaneous production of SA occurred (Fig. 4C and D). Although, these 272 activities of $\mathrm{AO} 7$ reduction and $\mathrm{SA}$ production were somewhat greater for the 273 biocathode in comparison to the abiotic cathode, indicating that the cells were 274 enhancing the AO7 reduction while using electrons provided from the cathode. 275 However, in comparison to Group I (Fig. 4A and B), the AO7 reduction efficiencies 276 under the Group II conditions were much lower. For example, 93.6 $\pm 1.0 \%$ of AO7 was 277 reduced in the biocathode without lactate within 120 h (Fig. 4C), while $95.16 \pm 0.59 \%$ of $278 \mathrm{AO} 7$ was reduced in the biocathode in the presence of $10 \mathrm{mM}$ lacate within $46 \mathrm{~h}$ (Fig. 279 4A). Consequently, it was evident that lactate was also important for providing 280 electrons for the BES biocathode reduction of AO7.

In BES hydrogen gas can be produced at the cathode and this can facilitate electron 283 transfer from the electrode to the bacteria (Croese et al. 2011). Experiments were 284 conducted to determine whether $\mathrm{H}_{2}$ was influencing the AO7 reduction (Group III). 285 These were performed by controlling the cathode potential at $-408 \mathrm{mV}$ (vs. SHE), at which hydrogen gas was not produced (Wang et al. 2011). In the absence of the external 
287 electron donor and the possible electron facilitator, $\mathrm{H}_{2}$, in the biocathode, $\mathrm{AO} 7$ 288 reduction was faster than that of in the abiotic cathode (Fig. 4E). Thus, indicating that $S$. 289 oneidensis could still enhance the reduction of AO7 without the electron facilitator. At $290120 \mathrm{~h}$, the AO7 reduction efficiency of the biocathode in the Group III experiment was 291 around $92.1 \pm 0.3 \%$ (Fig. 4E), which was close to that of $93.6 \pm 1.0 \%$ in the biocathode in 292 Group II (Fig. 4C), demonstrating that $\mathrm{H}_{2}$ had a negligible effect on AO7 reduction by 293 S. oneidensis. Additionally, the production profiles of SA (Fig. 4F) were in good 294 agreement with the reduction profiles of AO7 (Fig. 4E) and supported the conclusions 295 drawn here. It was also seen that when the cathode hydrogen production was limited, 296 the current generation of the biocathode was higher than that of the abiotic cathode (Fig. 297 3C), this further supporting that $S$. oneidensis could accelerate the AO7 reduction in the absence of the electron transfer facilitator.

\subsection{Role of S. oneidensis in the biocathodic reduction of AO7}

$301 \mathrm{CV}$ analysis of the closed biocathode and abiotic cathode in the presence of AO7 was 302 performed (Fig. 5). In comparison to the CV profile of the abiotic cathode, the profile of the closed circuit biocathode has more positive onset potentials together with greater 304 levels of cathodic current (Figure 5). This demonstrates that the AO7 reduction by $S$. 305 oneidensis contributed to the enhanced current. The AO7 reduction peaks occurred at 306 about $-0.66 \mathrm{~V}$ for the closed circuit biocathodes and at about $-0.75 \mathrm{~V}$ for the abiotic cathode (Fig. 5). The reduction peak of the closed circuit biocathode with lactate was higher than that of the closed circuit biocathode without lactate, thus indicating that the 309 presence of the external electron donor facilitated the AO7 reduction. It has been 310 reported that the biodegradation of AO7 was enhanced by S. oneidensis through co- 
311 metabolism with the addition of pyruvate in microbial fuel cell (Fernando et al. 2012).

312 Thus there is a possibility that AO7 could be co-metabolically reduced by $S$. oneidensis

313 MR-1 through the addition of lactate. Moreover, the first-order kinetic constant of AO7

314 reduction decreased from around 0.25 to about 0.13 from when lactate is present to 315 absent.

317 It has been proposed that these redox peaks result from the reversible reaction of the

318 aromatic amine intermediates that form from the AO7 reduction (Mu et al. 2009, Kong 319 et al. 2014). The variation in CV profiles on the closed circuit biocathode and abiotic 320 cathode indicate that the electron transfer pathways are different, and this has been 321 observed previously (Kong et al. 2014). From the CVs lower over-potentials for the 322 AO7 reduction at the closed circuit biocathode were evident in comparison with that of 323 the abiotic cathode, regardless of the presence of lactate. This also supports that the 324 closed circuit biocathode was more efficiently catalyzing the AO7 reduction in 325 comparison to the abiotic cathode.

\section{4. Conclusions}

328 This work demonstrated that the S. oneidensis formed biocathode could efficiently 329 enhance the reduction of AO7 with the production of SA. Pure culture formed 330 biocathode could offer an opportunity for unraveling the unclear electron transfer 331 mechanisms between the electrode and microorganisms. Therefore, this study provides 332 fundamental knowledge of the interaction between the cathodic electrode and 333 microorganisms, thus benefiting the understanding of biocathodic electron transfer 334 pathways and corresponding molecular mechanisms. 


\section{Acknowledgement}

337 We gratefully acknowledge the financial support by National Science Foundation for 338 Distinguished Young Scholars (Grant No. 51225802), the National Natural Science 339 Foundation of China (No. 31500084), the State Key Laboratory of Urban Water 340 Resource and Environment of HIT (No. 2016DX03) and the Hundred Talents Program of the Chinese Academy of Sciences (No. 29BR2013001).

\section{References}

Bay, H.H., Lim, C.K., Kee, T.C., Ware, I., Chan, G.F., Shahir, S., and Ibrahim, Z. (2014) Decolourisation of acid orange 7 recalcitrant auto-oxidation coloured byproducts using an acclimatised mixed bacterial culture. Environ Sci Pollut Res Int 21: 3891-3906.

Belchik, S.M., Kennedy, D.W., Dohnalkova, A.C., Wang, Y., Sevinc, P.C., Wu, H., et al. (2011) Extracellular reduction of hexavalent chromium by cytochromes MtrC and OmcA of Shewanella oneidensis MR-1. Appl Environ Microbiol 77: 40354041.

Cai, P.J., Xiao, X., He, Y.R., Li, W.W., Chu, J., Wu, C., et al. (2012) Anaerobic biodecolorization mechanism of methyl orange by Shewanella oneidensis MR-1. Appl Microbiol Biotechnol 93: 1769-1776.

Cerniglia, K.-T.C.A.C.E. (1992) Mutagenicity of azo dyes: Structure-activity relationships. Mutation Research 277: 201-220.

Croese, E., Pereira, M.A., Euverink, G.J., Stams, A.J., and Geelhoed, J.S. (2011) Analysis of the microbial community of the biocathode of a hydrogen-producing microbial electrolysis cell. Appl Microbiol Biotechnol 92: 1083-1093.

dos Santos, A.B., Traverse, J., Cervantes, F.J., and van Lier, J.B. (2005) Enhancing the electron transfer capacity and subsequent color removal in bioreactors by applying 

52.

Elisangela, F., Andrea, Z., Fabio, D.G., de Menezes Cristiano, R., Regina, D.L., Artur, C.-P. (2009) Biodegradation of textile azo dyes by a facultative Staphylococcus arlettae strain $\mathrm{VN}-11$ using a sequential microaerophilic/aerobic process. Int Biodeter Biodegr 63: 280-288.

Feng, H., Zhang, X., Guo, K., Vaiopoulou, E., Shen, D., Long, Y., et al. (2015) 370 Electrical stimulation improves microbial salinity resistance and organofluorine removal in bioelectrochemical systems. Appl Environ Microbiol 81: 3737-3744.

Fernando, E., Keshavarz, T., Kyazze, G. 2012. Enhanced bio-decolourisation of acid orange 7 by Shewanella oneidensis through co-metabolism in a microbial fuel cell. Int Biodeter Biodegr 72: 1-9.

Hong, Y., Xu, M., Guo, J., Xu, Z., Chen, X., and Sun, G. (2007) Respiration and growth of Shewanella decolorationis S12 with an azo compound as the sole electron acceptor. Appl Environ Microbiol 73: 64-72.

Hong, Y.G., and Gu, J.D. (2010) Physiology and biochemistry of reduction of azo compounds by Shewanella strains relevant to electron transport chain. Appl Microbiol Biotechnol 88: 637-643.

Kong, F., Wang, A., Cheng, H., and Liang, B. (2014) Accelerated decolorization of azo dye Congo red in a combined bioanode--biocathode bioelectrochemical system with modified electrodes deployment. Bioresour. Technol. 151: 332-339.

Kong, F., Wang, A., Ren, H.-Y., Huang, L., Xu, M., and Tao, H. (2014) Improved dechlorination and mineralization of 4-chlorophenol in a sequential biocathode-bioanode bioelectrochemical system with mixed photosynthetic bacteria. Bioresour. Technol. 158: 32-38.

Liang, B., Cheng, H., Van Nostrand, J.D., Ma, J., Yu, H., Kong, D., et al. (2014) Microbial community structure and function of nitrobenzene reduction biocathode in response to carbon source switchover. Water Res 54C: 137-148.

Liang, B., Cheng, H.Y., Kong, D.Y., Gao, S.H., Sun, F., Cui, D., et al. (2013) Accelerated reduction of chlorinated nitroaromatic antibiotic chloramphenicol by biocathode. Environ Sci Technol 47: 5353-5361. 
Liu, S., Song, H., Wei, S., Liu, Q., Li, X., and Qian, X. (2015) Effect of direct electrical stimulation on decolorization and degradation of azo dye reactive brilliant red $\mathrm{X}$ 3B in biofilm-electrode reactors. Biochem. Eng. J. 93: 294-302.

Lončar, N., Janović, B., Vujčić, M., Vujčić, Z. 2012. Decolorization of textile dyes and effluents using potato (Solanum tuberosum) phenoloxidase. Int Biodeter Biodegr 72: 42-45.

Mu, Y., Rabaey, K., Rozendal, R.A., Yuan, Z., and Keller, J. (2009) Decolorization of azo dyes in bioelectrochemical systems. Environ Sci Technol 43: 5137-5143.

Pandey, A., Singh, P., Iyengar, L. 2007. Bacterial decolorization and degradation of azo dyes. Int Biodeter Biodegr 59: 73-84.

Pearce, C.I., Christie, R., Boothman, C., von Canstein, H., Guthrie, J.T., and Lloyd, J.R. (2006) Reactive azo dye reduction by Shewanella strain J18 143. Biotechnol Bioeng 95: 692-703.

Perreault, N.N., Crocker, F.H., Indest, K.J., and Hawari, J. (2012) Involvement of cytochrome c CymA in the anaerobic metabolism of RDX by Shewanella oneidensis MR-1. Can J Microbiol 58: 124-131.

Rosenbaum, M., Aulenta, F., Villano, M., and Angenent, L.T. (2011) Cathodes as electron donors for microbial metabolism: which extracellular electron transfer mechanisms are involved? Bioresour Technol 102: 324-333.

Rosenbaum, M.A., Bar, H.Y., Beg, Q.K., Segre, D., Booth, J., Cotta, M.A., and Angenent, L.T. (2012) Transcriptional analysis of Shewanella oneidensis MR-1 with an electrode compared to $\mathrm{Fe}(\mathrm{III})$ citrate or oxygen as terminal electron acceptor. PLoS One 7: e30827.

Singh, R.L., Singh, P.K., Singh, R.P. 2015. Enzymatic decolorization and degradation of azo dyes--A review. Int Biodeter Biodegr 104: 21-31.

Tan, L., Ning, S., Xia, H., Sun, J. (2013). Aerobic decolorization and mineralization of azo dyes by a microbial community in the absence of an external carbon source. Int Biodeter Biodegr 85, 210-216.

Tan, N.C., van Leeuwen, A., van Voorthuizen, E.M., Slenders, P., Prenafeta-Boldú, F.X., Temmink, H., et al. (2005) Fate and biodegradability of sulfonated aromatic amines. Biodegradation 16: 527-537. 
425 Tony, B.D., Goyal, D., Khanna, S. (2009) Decolorization of textile azo dyes by aerobic 426 bacterial consortium. Int Biodeter Biodegr 63: 462-469.

427 Van der Zee, F.P., and Villaverde, S. (2005) Combined anaerobic--aerobic treatment of 428 azo dyesa short review of bioreactor studies. Water Res 39: 1425-1440.

429 Wang, A.J., Cheng, H.Y., Liang, B., Ren, N.Q., Cui, D., Lin, N., et al. (2011) Efficient 430 reduction of nitrobenzene to aniline with a biocatalyzed cathode. Environ Sci 431 Technol 45: 10186-10193.

432 Wang, H., Luo, H., Fallgren, P.H., Jin, S., and Ren, Z.J. (2015) Bioelectrochemical 433 system platform for sustainable environmental remediation and energy generation. $434 \quad$ Biotechnol Adv 33: 317-334.

435 Wang, Y.Z., Wang, A.J., Zhou, A.J., Liu, W.Z., Huang, L.P., Xu, M.Y., and Tao, H.C. 436 (2014) Electrode as sole electrons donor for enhancing decolorization of azo dye 437 by an isolated Pseudomonas sp. WYZ-2. Bioresour Technol 152: 530-533.

438 Xiao, X., Xu, C.C., Wu, Y.M., Cai, P.J., Li, W.W., Du, D.L., and Yu, H.Q. (2012) 439 Biodecolorization of naphthol green B dye by Shewanella oneidensis MR-1 under $440 \quad$ anaerobic conditions. Bioresour Technol 110: 86-90.

441 Xie, G.-J., Liu, B.-F., Ding, J., Ren, H.-Y., Xing, D.-F., and Ren, N.-Q. (2011) $442 \quad$ Hydrogen production by photo-fermentative bacteria immobilized on fluidized bio443 carrier. Int J Hydrogen Energ 36: 13991-13996.

444 Yang, Y., Xiang, Y., Sun, G., Wu, W.M., and Xu, M. (2015) Electron acceptor445 dependent respiratory and physiological stratifications in biofilms. Environ Sci 446 Technol 49: 196-202. 
Table 1. Kinetic constants of AO7 reduction and SA production in the presence and absence of lactate

\begin{tabular}{|c|c|c|c|c|c|c|}
\hline $\begin{array}{ll}\text { Initial } & \mathrm{AO} 7 \\
(\mathrm{mM}) & \end{array}$ & concentration & $\begin{array}{l}\text { Kinetics } \\
\text { constant of } \\
\text { AO7 reduction } \\
(\kappa)\end{array}$ & $\begin{array}{l}\text { Correlation } \\
\text { coefficient } \\
\text { of AO7 } \\
\text { reduction } \\
\left(\mathrm{R}^{2}\right)\end{array}$ & $\begin{array}{l}\text { Final SA } \\
\text { production } \\
\text { concentration } \\
(\mathrm{mM})\end{array}$ & $\begin{array}{l}\text { Kinetics } \\
\text { constant of SA } \\
\text { production } \\
(\kappa)\end{array}$ & $\begin{array}{l}\text { Correlation } \\
\text { coefficient } \\
\text { of SA } \\
\text { production } \\
\left(\mathrm{R}^{2}\right)\end{array}$ \\
\hline \multicolumn{7}{|c|}{ In the presence of lactate } \\
\hline $\begin{array}{l}\text { Closed } \\
\text { circuit } \\
\text { biocathode }\end{array}$ & $0.2485 \pm 0.0038$ & $0.063 \pm 0.002$ & 0.9986 & $0.2565 \pm 0.0075$ & $0.0503 \pm 0.004$ & 0.994 \\
\hline Abiocathode & $0.2531 \pm 0.0010$ & $0.0272 \pm 0.0027$ & 0.990 & $0.2351 \pm 0.0095$ & $0.0171 \pm 0.0020$ & 0.993 \\
\hline $\begin{array}{l}\text { Open circuit } \\
\text { biocathode }\end{array}$ & $0.2500 \pm 0.0089$ & $0.0239 \pm 0.0022$ & 0.984 & $1.4561 \pm 2.0663$ & $0.0021 \pm 0.0032$ & 0.986 \\
\hline \multicolumn{7}{|c|}{ In the absence of lactate } \\
\hline $\begin{array}{l}\text { Closed } \\
\text { circuit } \\
\text { biocathode }\end{array}$ & $0.1301 \pm 0.0049$ & $0.0479 \pm 0.0035$ & 0.9914 & $0.1254 \pm 0.0022$ & $0.0467 \pm 0.0025$ & 0.9973 \\
\hline Abiocathode & $0.1286 \pm 0.0039$ & $0.0324 \pm 0.002$ & 0.9937 & $0.1050 \pm 0.0041$ & $0.0396 \pm 0.0043$ & 0.9901 \\
\hline
\end{tabular}




\section{Figure Captions IBB-D-16-00431}

Figure 1. Current change during the biofilm formation of S. oneidensis on the BES cathodic electrode prior to use in the batch tests (A), and detection of $\mathrm{AO} 7$ in the cathode was evident only during the batch experiment (absorbance at $484 \mathrm{~nm})(B)$.

Figure 2. Scanning electron microscopy (SEM) micrographs of the carbon cloth cathode in the BES after the $S$. oneidensis biofilm formation period, grown in the absence of lactate; at 10000 times (A) and 2000 times (B) amplification.

Figure 3. Cathodic potentials and current generation with lactate at the closed circuit biocathode (A), without lactate at the closed circuit biocathode (B), and when the cathode potential was controlled at -408 $\mathrm{mV}$ without lactate (vs SHE) to limit hydrogen gas production (C).

Figure 4. Reduction of $\mathrm{AO} 7$ and production of SA under different operational conditions in the batch BES tests: in the presence of lactate in three different operational modes (biocathode, opended circuit biocathode, abiotic cathode) (A and B); in the absence of lactate in two different operational modes (biocathode and abiotic cathode) (C and D); and in the absence of lactate when cathode potential was controlled at $-408 \mathrm{mV}$ (vs SHE) in two different operational modes (biocathode and abiotic cathode) (E and F).

Figure 5. Cyclic voltammograms of the BES biocathode with or without lactate, and of the abiotic cathode. 

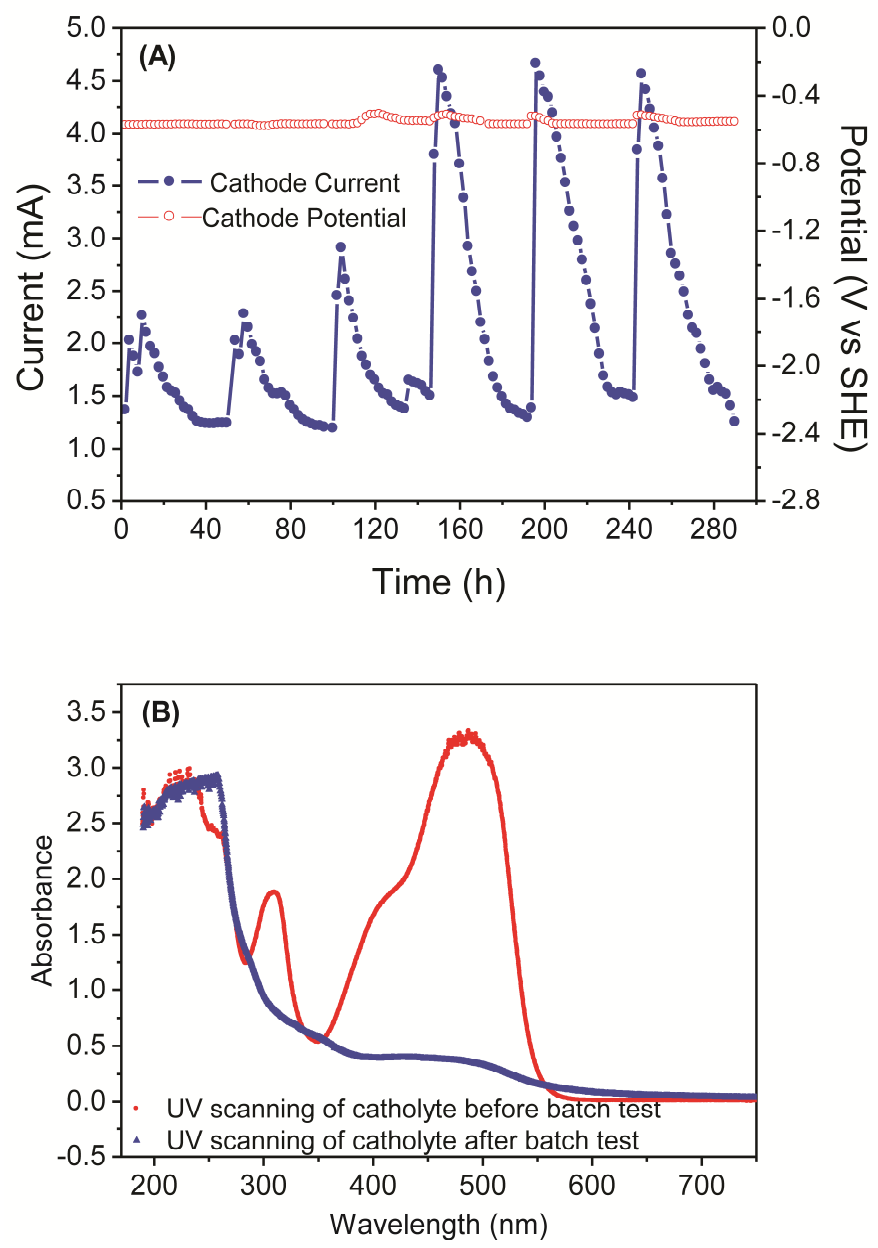


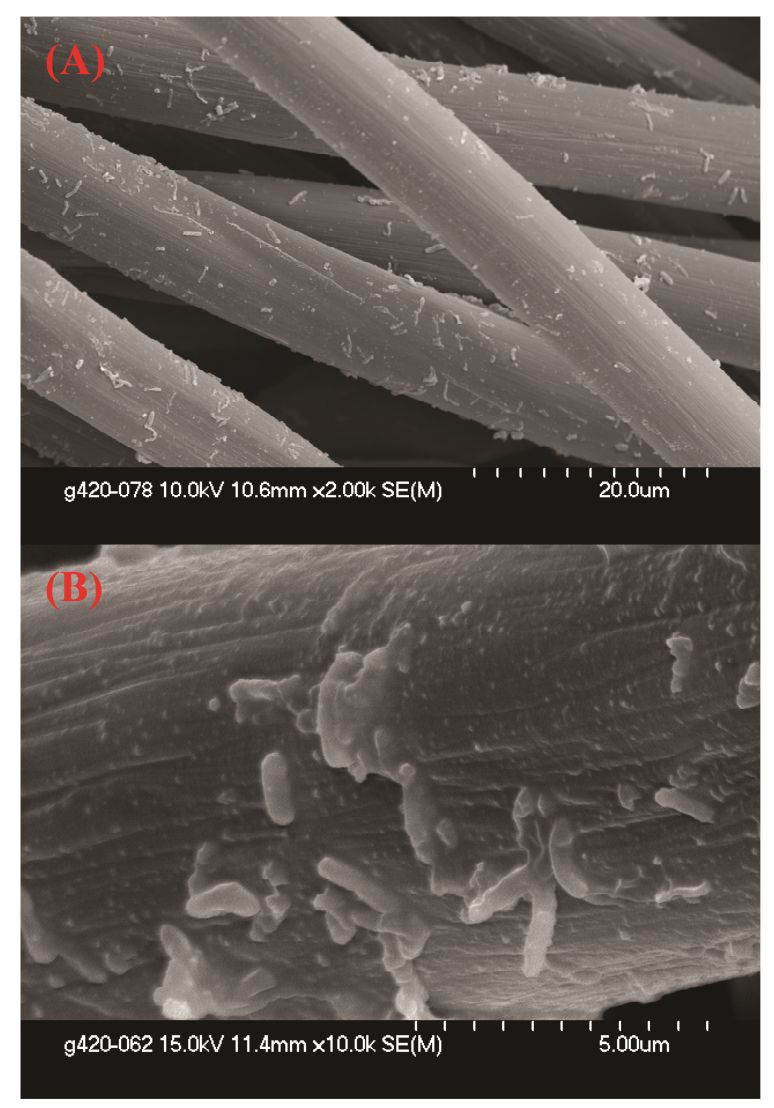



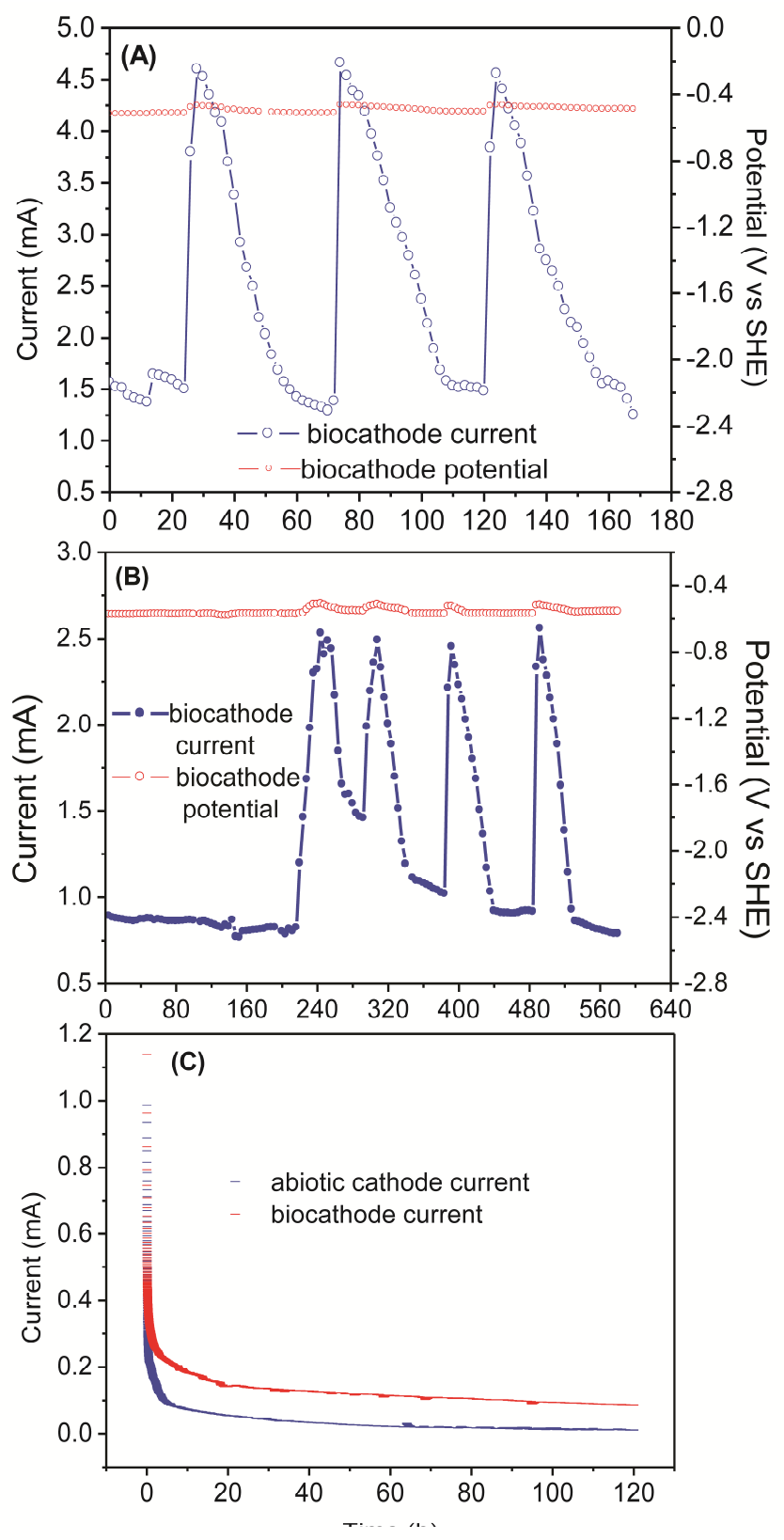


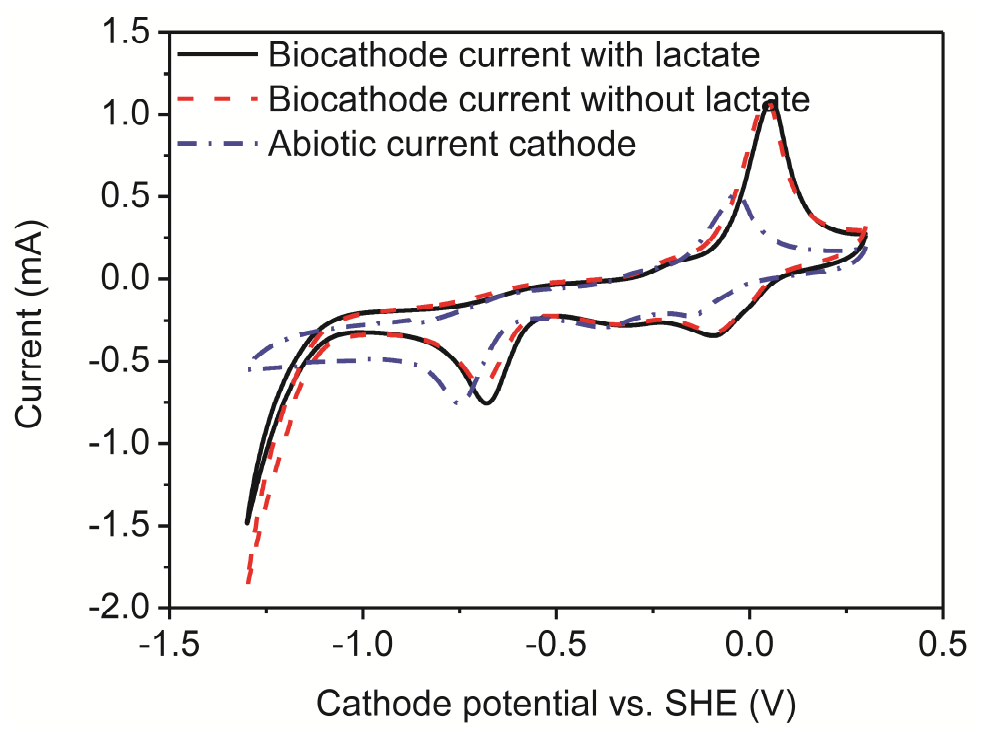

